\title{
Classical novae in outburst: The early evolution of the ultraviolet continuum
}

\author{
A. Cassatella ${ }^{1,2}$, A. Altamore ${ }^{2}$, and R. González-Riestra ${ }^{3}$ \\ 1 CNR, Istituto di Astrofisica Spaziale, via del Fosso del Cavaliere 100, 00133 Rome, Italy \\ 2 Dipartimento di Fisica E. Amaldi, Università Roma Tre, via della Vasca Navale 84, 00146 Rome, Italy \\ 3 Laboratorio de Astrofísica Espacial y Física Fundamental, PO Box 50727, 28080 Madrid, Spain
}

Received 3 December 2001 / Accepted 15 January 2002

\begin{abstract}
In the framework of a phenomenological study of the ultraviolet properties of classical novae in outburst, we have selected 12 objects among the best monitored at low resolution with the IUE satellite, and studied the temporal evolution of the ultraviolet continuum and of the O I $1300 \AA$ line flux during the early post-outburst phase. We confirm that the UV flux maximum takes place systematically after the visual maximum and that its time delay is a linear function of $t_{3}$. A linear dependence on $t_{3}$ is also found for the duration of the UV outburst and for the time the $\mathrm{O}$ I line flux reaches a maximum. This latter time marks the start of the transition phase to nebular conditions. Within the uncertainties imposed by the sample of objects used and by the observational errors, these results suggest a quite homogeneous behaviour of classical novae in the ultraviolet range.
\end{abstract}

Key words. stars: novae, cataclysmic variables - techniques: spectroscopic - ultraviolet: stars

\section{Introduction}

Ultraviolet studies of individual classical novae in outburst have allowed important progress to be made in the understanding of the physics of the nova phenomenon (see González-Riestra \& Krautter 1998, for a recent review).

Thanks to the presence in the UV range of many emission lines arising from astrophysically important elements such as He, C, N, O, Mg, Ne etc., mostly observed in different ionization stages, it has been possible to determine the chemical abundances of the ejecta with an unprecedented accuracy (cf. Stickland et al. 1981 for V1668 Cyg; Williams et al. 1985; Vanlandingham et al. 1997 for V693 CrA; Schwartz et al. 1997a for PW Vul; Andreä et al. 1994 for several novae).

Based on these results it has been possible to refine our knowledge on the nucleosynthesis processes taking place during the outburst and on the nature of the underlying white dwarf. In particular, the detection of very high overabundances of Neon in some novae (the first one discovered was V693 CrA by Williams et al. 1985) led to the suggestion that the nova progenitor must be a O-Ne$\mathrm{Mg}$ white dwarf (Starrfield et al. 1986), in agreement with the theoretical scenario of Law and Ritter (1983), who

Send offprint requests to: A. Cassatella, e-mail: cassatella@fis.uniroma3.it predicted the existence of high mass white dwarfs resulting from the evolution of stars with initial mass in the range $8-10 M_{\odot}$.

In some cases, as those of V1668 Cyg (Stickland et al. 1981), V1370 Aql (Snijders et al. 1987), V1974 Cyg (Shore et al. 1994a), LMC 1998 \#1 (Schwartz et al. 1998) and LMC 1991 (Schwartz et al. 2001), it has been possible to combine $I U E$ observations with simultaneous ground based observations in the optical and infrared ranges. These data were essential to estimate the overall energetics of the nova outburst and the time evolution of the bolometric light curve, an information that provides important constraints for thermonuclear runaway models.

During its lifetime (1978-1996), the IUE satellite has observed 36 classical novae in outburst, obtaining nearly 1600 high and low resolution spectra. About $70 \%$ of the observations are concentrated on 12 objects, and provide a good coverage of all relevant phases from the outburst to the late decline.

In this paper, we carry out a systematic analysis of the low resolution spectra of these well monitored objects. We look at this sample as a whole, with the specific purpose to identify the general patterns, to establish correlations between observational parameters and to identify possible peculiarities. 
The observations (about 650 low resolution spectra) are presented in Sect. 2. In Sect. 3 we compare the visual light curve of six novae with the flux variations in two continuum bands around $1455 \AA$ and $2885 \AA$ and in the O I $1300 \AA$.

In Sect. 4 we discuss the ultraviolet light curves at $1455 \AA$ for the complete sample of 12 novae and provide an analytical relationship between the delay of the $1455 \AA$ flux maximum and the $t_{3}$ decay time. An analogous correlation is found between the duration of the outburst and $t_{3}$. The conclusions are presented in Sect. 5 .

\section{Observations}

\subsection{The IUE observations}

In this paper we report on the analysis of the IUE low resolution large-aperture spectra of 12 classical novae, selected as being as more closely followed by the IUE satellite from the outburst to the late decline phases. The spectra (about 650), were retrieved from the INES (IUE Newly Extracted Spectra) system through its Principal Centre at http://ines.laeff.esa.es, which provides also a detailed observing log. A full description of the INES system is given in Rodríguez-Pascual et al. (1999), Cassatella et al. (2000) and González-Riestra et al. (2000, 2001). A great care has been taken as to consider only measurements arising from spectra or portions of spectra well exposed and free of instrumental effects (in particular those arising from saturation).

\subsection{The novae sample}

The relevant information on the 12 objects is given in Table 1 which contains, for each nova, the day of the discovery $t_{\mathrm{disc}}$ and the time of the visual maximum $t_{0}$, the visual magnitude at maximum light $V_{\max }$, the decay time $t_{3}$ with the corresponding estimated error, and the $E(B-V)$ colour excess. References for the adopted values of $t_{3}$ (defined as the time to fade $3 \mathrm{mag}$ from visual maximum) and of $E(B-V)$ are given as footnotes to Table 1. Table 1 contains also the results of our measurements, and namely: the time delay of the UV maximum at $1455 \AA$ with respect to the visual maximum $\left(\Delta t_{1455}\right)$, the duration of the outburst in the $1455 \AA$ band $\left(\Delta T_{\text {outb. }}^{1455}\right)$, and the time delay of the flux maximum in the $\mathrm{O}$ I line at $1300 \AA$ with respect to the visual maximum $(\Delta t(\mathrm{O} \mathrm{I}))$. The latter three parameters, given together with their corresponding errors, are discussed further in Sects. 3 and 4. Interesting novae such as GQ Mus and QU Vul were not included in the present sample, because the IUE observations, although quite abundant, did not cover well enough the specific aspects studied here.

Our sample includes objects showing chemical or physical peculiarities. An enhancement of Neon and heavier elements has been reported for V693 CrA, V351 Pup, V1974 Cyg and V838 Her (see González-Riestra \& Krautter 1998, and references therein). ROSAT observations have shown V1974 Cyg, V351 Pup and V838 Her to be hard $\mathrm{X}$-ray sources in the few days-months after the outburst (Orio et al. 2001 and references therein). V1974 Cyg showed also a super-soft spectrum from 6 to 21 months after the outburst (Krautter et al. 1996). V705 Cas underwent a strong episode of dust formation about two months after the outburst (Shore et al. 1994b). Dust formation has also been observed in Nova LMC 1988 \#1 (Schwartz et al. 1998). In both cases, only data presumably not distorted by dust formation have been considered.

\subsection{The $t_{3}$ values}

The objects in Table 1 cover a wide range of speed class from the fast novae V838 Her 1991 and N LMC 1991, to the slow novae V 705 Cas 1993 and PW Vul 1994\#2. The optical decay time $t_{3}$ is a key parameter for our study, although the determinations available from the literature are sometimes uncertain. In the case of V705 Cas, only a very rough evaluation of $t_{3}$ can be made because the star suffered from a substantial drop in magnitude due to dust formation between day 62 (Evans et al. 1996) and 71 (Mason et al. 1998): the $t_{3}$ value here adopted has been obtained by extrapolating the visual light curve provided by Evans et al. (1996). The uncertainty on the $t_{3}$ time for PW Vul is discussed by Robb \& Scarf (1995). For Nova LMC 1992, the only information available is from the IAU circulars and from the Fine Error Sensor (FES) on board IUE. In the case of Nova LMC 1991, $t_{3}$ was obtained by extrapolation of the visual light curve from Della Valle (1991). The errors on $t_{3}$ in Table 1 were estimated by us on the basis of the photometric data available.

\section{Evolution of the ultraviolet continuum and of the OI $1300 \AA$ A line}

In Fig. 1 we show the time evolution of the visual magnitude, the $2885 \AA$ and $1455 \AA$ fluxes, the $C(1455-2885)$ colour index defined below, and the flux of the O I $1300 \AA$ line for the following novae: V838 Her 1991, N LMC 1992, V351 Pup, V1668 Cyg, N LMC 1988a, and V1974 Cyg. As abscissae we have used the normalized time $\left(t-t_{0}\right) / t_{3}$, being $t$ the time of the observations and $t_{0}$ the time of the visual maximum.

The figure shows that, with the adopted normalization of the time scale to $t_{3}$, the photometric behaviour of the six novae is remarkably similar, in spite of the wide range of $t_{3}$ values covered, from 5 days for V838 Her to 42 days for V1794 Cyg.

The ultraviolet continuum has been measured in two narrow bands, $20 \AA$ wide, centered at 1455 and $2885 \AA$, taken to avoid strong emission or absorption lines. Let us define the following ultraviolet colour index:

$$
C(1455-2885)=-2.5 \log (F(1455 \AA) / F(2885 \AA)
$$

where $F(1455 \AA)$ and $F(2885 \AA)$ are the corresponding mean reddening-corrected fluxes. The reddening correction has been made using the $E(B-V)$ values in Table 1 

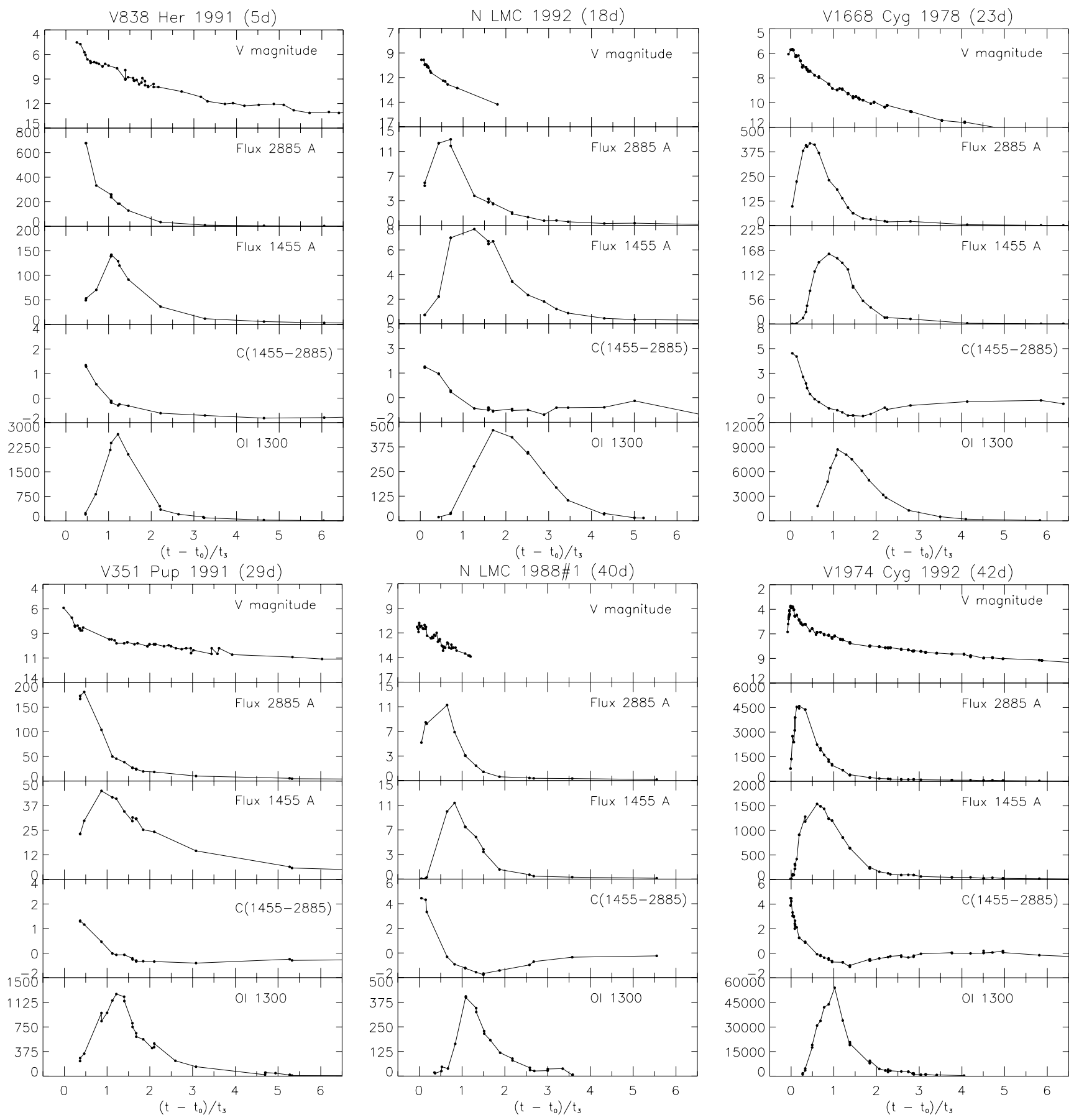

Fig. 1. For six novae in our sample we show the time evolution of the $V$ magnitude, the observed fluxes in two narrow continuum bands centered at $2885 \AA$ and $1455 \AA$, the reddening-corrected ultraviolet colour index $C(2885-1455)$, and the observed flux in the OI $1300 \AA$ emission line. Abscissae give $\left(t-t_{0}\right) / t_{3}$, i.e. the time elapsed after the outburst, normalized to $t_{3}$. Note how, after this normalization, the curves are remarkably similar. The $1455 \AA$ and $2885 \AA$ fluxes are in units of $10^{-13} \mathrm{erg} \mathrm{cm}^{-2} \mathrm{~s}^{-1} \mathrm{~A}^{-1}$; the OI fluxes are in units of $10^{-13} \mathrm{erg} \mathrm{cm}^{-2} \mathrm{~s}^{-1}$.

which include, for the LMC novae, a galactic foreground colour excess $E(B-V)_{\mathrm{G}}=0.05$. For the Galactic and LMC extinction laws we have used Seaton (1979) and Fitzpatrick (1986), respectively, together with $R=3.1$.

The behaviour of the $C(1455-2885)$ colour index is quite homogeneous for the six novae, as it can be easily recognized from Fig. 1: typical of a red object in the earliest phases, it becomes rapidly hotter and hotter to reach a local minimum, followed by an upturn to redder colours in the case of V1668 Cyg, N LMC 1988\#1 and V1974 Cyg, or showing a colour plateau, as in V351 Pup, V838 Her and N LMC 1992. The upturn to redder colours or the plateau starts to appear when $\left(t-t_{0}\right) \approx 1.5 t_{3}$. At this time $C(1455-2885) \approx-1.1 \pm 0.8$, which would correspond to a colur temperature of about $21000 \mathrm{~K}$. 
Table 1. Basic parameters of novae.

\begin{tabular}{lcrrrrrrl}
\hline \hline Nova & $t_{\text {disc. }}$ & $t_{0}$ & $V_{\text {max }}$ & \multicolumn{1}{c}{$t_{3}$} & $\Delta t_{1455}$ & $\Delta T_{\text {outb. }}^{1455}$ & $\Delta t(\mathrm{O}$ I $)$ & $E(B-V)$ \\
\hline V1668 Cyg 1978 & 3762 & 3763 & 6.1 & $23 \pm 3^{1}$ & $27 \pm 3$ & $30 \pm 3$ & $36 \pm 5$ & $0.4 \pm 0.1^{13}$ \\
V 693 CrA 1981 & 4697 & 4697 & 6.5 & $12 \pm 2^{2}$ & $12 \pm 3$ & $9 \pm 5$ & $11 \pm 2$ & $0.2 \pm 0.1^{14}$ \\
PW Vul 1984 & 5909 & 5917 & 6.4 & $100 \pm 20^{3}$ & $79 \pm 10$ & $100 \pm 6$ & $96 \pm 20$ & $0.55 \pm 0.1^{15}$ \\
OS And 1986 & 6770 & 6773 & 6.2 & $20 \pm 3^{4}$ & $19 \pm 5$ & $17 \pm 6$ & $27 \pm 20$ & $0.25 \pm 0.05^{4}$ \\
N LMC 1988\#1 & 7242 & 7244 & 10.7 & $40 \pm 2^{5}$ & $33 \pm 4$ & $46 \pm 6$ & $42 \pm 10$ & $0.15 \pm 0.05^{5}$ \\
N LMC 1988\#2 & 7747 & 7748 & 10.3 & $10 \pm 3^{6}$ & $7 \pm 3$ & $18 \pm 5$ & & $0.15 \pm 0.05^{18}$ \\
V838 Her 1991 & 8339 & 8339 & 5.0 & $5 \pm 1^{7}$ & $5 \pm 1$ & $5 \pm 3$ & $7 \pm 2$ & $0.56 \pm 0.05^{7}$ \\
N LMC 1991 & 8365 & 8372 & 8.8 & $8 \pm 3^{8}$ & $10 \pm 5$ & $21 \pm 5$ & $4 \pm 2$ & $0.15 \pm 0.05^{18}$ \\
V351 Pup 1991 & 8618 & 8618 & 6.4 & $29 \pm 5^{9}$ & $25 \pm 2$ & $38 \pm 5$ & $34 \pm 4$ & $0.3 \pm 0.1^{16}$ \\
V1974 Cyg 1992 & 8672 & 8675 & 4.4 & $42 \pm 3^{10}$ & $26 \pm 2$ & $40 \pm 5$ & $42 \pm 7$ & $0.36 \pm 0.04^{17}$ \\
N LMC 1992 & 8937 & 8938 & 10.2 & $18 \pm 3^{11}$ & $18 \pm 3$ & $26 \pm 4$ & $32 \pm 8$ & $0.15 \pm 0.05^{18}$ \\
V705 Cas 1993 & 9329 & 9333 & 6.6 & $90 \pm 15^{12}$ & $>65$ & $>30$ & $>61$ & $0.566^{19}$ \\
\hline
\end{tabular}

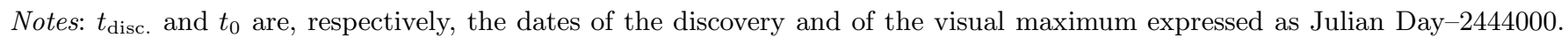
$\Delta t_{1455}$ is the time delay of the flux maximum at $1455 \AA$ with respect to the visual maximum (days). $\Delta T_{\text {outb. }}^{1455}$ is a measure of the duration of the outburst in the ultraviolet, in days (see text). References for $t_{3}$ : (1) Duerbeck et al. (1980) (see also Cassatella et al. 1979); (2) Caldwell (1982); (3) Gehrz et al. (1988); (4) Schwarz et al. (1997b); (5) Schwartz et al. (1998); (6) Sekiguchi et al. (1989); (7) Vanlandingham et al. (1996); (8) from the $V$ light curve in Della Valle (1991); (9) estimated by us from IAU circulars and FES photometry (see also light curve in Saizar et al. 1996); (10) Chochol et al. (1993); (11) estimated by us from IAU circulars and FES photometry; (12) Mason et al. (1998); (13) Stickland et al. (1981); (14) Vanlandingham et al. (1997); (15) Andreä et al. (1991); (16) Orio et al. (1996); (17) Austin et al. (1996); (18) estimated by us from IUE spectra; (19) Hric et al. (1998).

An interpretation of these results has been suggested by Cassatella and González-Riestra (1990). They pointed out that, while the envelope expands, unveiling the optically thick continuum from deeper and hotter layers, an increasing fraction of the envelope itself becomes ionized and eventually transparent to the Balmer continuum radiation which, at least in the optically thin case and for electron temperatures of the order of $10000 \mathrm{~K}$, is significantly stronger in the $2885 \AA$ band than in the $1455 \AA$ band. Its contribution can then cause an upturn to redder colours or a colour plateau, depending on the relative contribution of the expanding pseudo-photosphere and of the nebular continuum. The colour reversal or the colour plateau are then related to the time optically thin conditions start to set in.

This interpretation is supported by the time evolution of the flux from the O I $1300 \AA$ resonance triplet (UV 2), which is shown in the bottom panels of Fig. 1. It clearly appears that O I reaches a flux maximum somewhat earlier than the UV colour minimum, i.e. at $\left(t-t_{0}\right) / t_{3} \approx 1.2$. These lines are likely produced via the Bowen flourescence mechanism, by which the $3 \mathrm{~d}$ levels of O I are photoexcited by hydrogen Ly $\beta$ photons; a cascade decay process follows in which the upper level of the O I UV 2 resonance multiplet are populated, giving rise to the observed emission (Johansson \& Hamann 1993; Kastner \& Bhatia 1995 and references therein). The halt to the initial fast hardening of the ultraviolet continuum at $\left(t-t_{0}\right) \approx 1.5 t_{3}$ appears then to be related with the time the nova envelope starts to become optically thin in the Balmer continuum. After its peak emission, the $\mathrm{O}$ I line decreases steadily and falls near the detection limit for $\left(t-t_{0}\right) / t_{3} \geq 3$, due to oxygen ionization.

We have measured the delay of the O I flux maximum with respect to the visual maximum, $\Delta t(\mathrm{O} \mathrm{I})$, for the novae in Table 1 and plotted the results as a function of $t_{3}$ in Fig. 2. LMC 1988 \#2 was not included because not showing any measurable emission at that wavelength. As for V705 Cas, affected by dust formation, only a lower limit could be obtained, indicated in the figure. If the errors on both variables are taken into account (Press et al. 1992), a linear fit to all the data, except the latter two cases, provides:

$$
\Delta t(\mathrm{O} \text { I })=(0.36 \pm 2.35)+(1.17 \pm 0.14) t_{3} \quad(\text { days })
$$

\section{The time delay of the UV maximum and its duration}

In this section we study the time evolution of the $1455 \AA$ continuum for the 12 novae in Table 1 . The observed $1455 \AA$ fluxes are plotted as a function of the time after optical outburst in Fig. 3, where the objects are arranged in order of increasing $t_{3}$.

As shown in Fig. 3, the time delay of the UV maximum with respect to the visual maximum, $\Delta t_{1455}$, is an increasing function of $t_{3}$ (see also the preliminary results of Cassatella \& González-Riestra 1990). To quantify this 


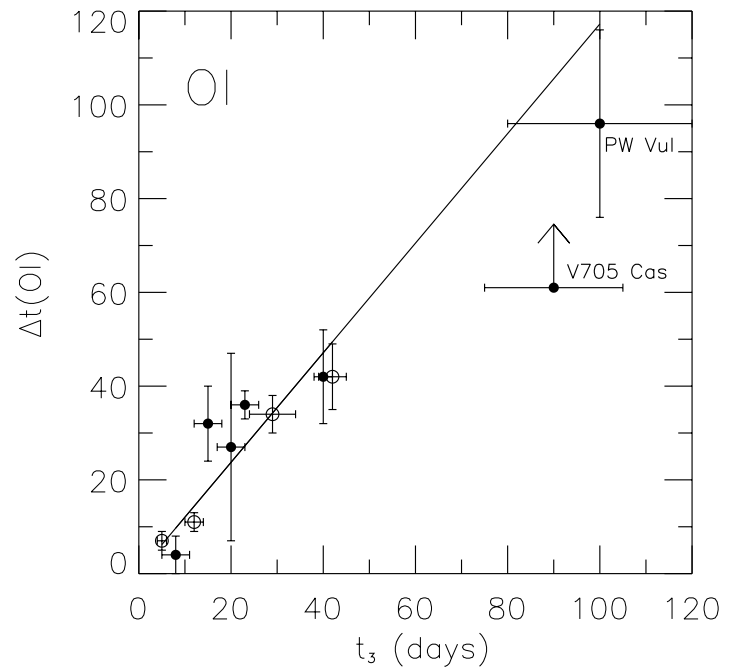

Fig. 2. The time of the O I $1300 \AA$ maximum, $\Delta t(\mathrm{O}$ I $)$, is plotted as a function of $t_{3}$ for the novae in Table 1 . Neon novae are indicated as open circles

feature, we have measured $\Delta t_{1455}$ for the 12 novae in Fig. 3 and plotted the results in Fig. 4 as a function of $t_{3}$.

A linear fit to the data provides:

$\Delta t_{1455}=(1.96 \pm 1.34)+(0.74 \pm 0.07) t_{3} \quad($ days $)$

Also in this case V705 Cas was not included in the fit because only a lower limit to the time delay could be obtained.

The above results can be understood by assuming that during a time of the order of $t_{3}$, a classical nova radiates at constant bolometric luminosity, as supported by theoretical work and multi-wavelength observations (see Introduction). Since the radius of the pseudo-photosphere shrinks as the outer envelope expands, its effective temperature steadily increases, causing a progressive shift of the emission peak to shorter and shorter wavelengths. This is observed as a progressive hardening of the UV continuum (see the decrease of the $C(1455-2885)$ colour index) and results in a systematic delay of UV continuum peak emission with respect to the visual band.

The second pattern which appears clearly from Fig. 3 is that the duration of the outburst in the $1455 \AA$ band is shorter for novae with faster evolution in the visual range. The only deviating object is V705 Cas, whose $1455 \AA$ flux is visibly depressed in coincidence with the quoted dust formation process (see Sect. 2). A quantitative estimate of the UV outburst duration has been obtained by measuring the full width at half maximum of the $1455 \AA$ light curve around its maximum. The values so obtained are plotted as a function of $t_{3}$ in Fig. 5. A linear regression to the data, not including V705 Cas, provides:

$$
\left.\Delta T_{\text {outb. }}^{1455}=(2.52 \pm 3.00)+(1.06 \pm 0.13) t_{3} \quad \text { (days }\right)
$$

\section{Conclusions and discussion}

The observed properties of classical novae in outburst are difficult to interpret because they depend, in an intricate

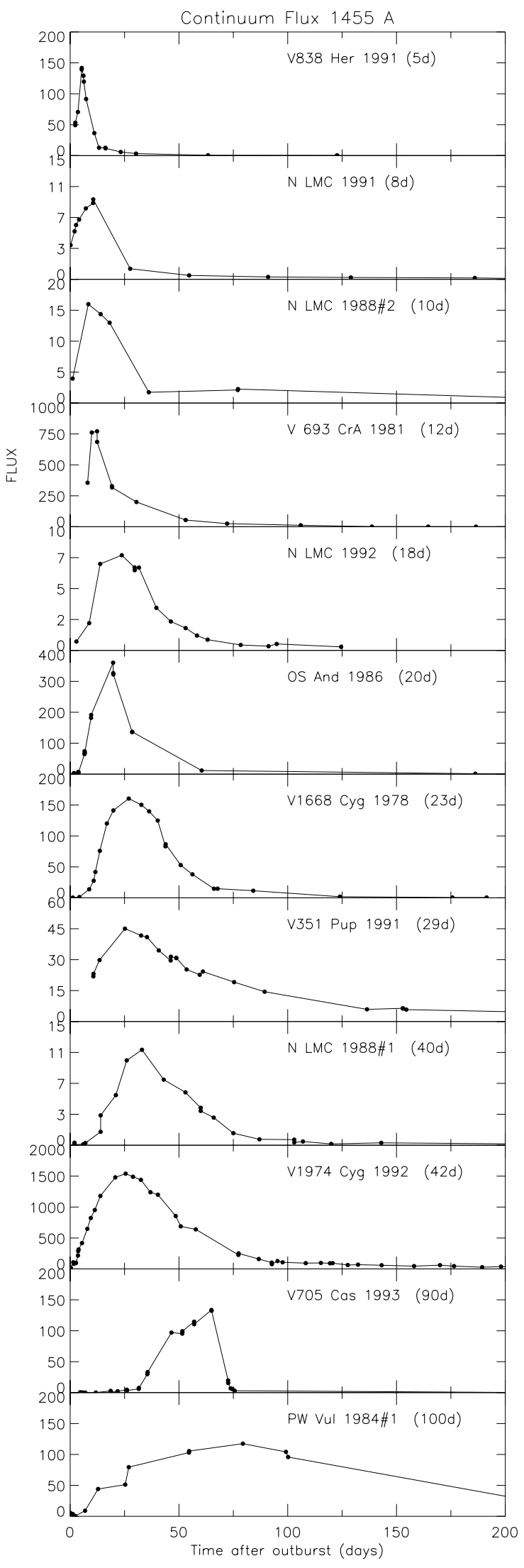

Fig. 3. Time evolution of the UV continuum at $1455 \AA$ for the novae in Table 1 . The $t_{3}$ times are indicated in brackets. Note the gradual increase of the UV outburst duration as $t_{3}$ increases and the anomalous behaviour of V705 Cas (see text). Fluxes are in the same units as in Fig. 1. 


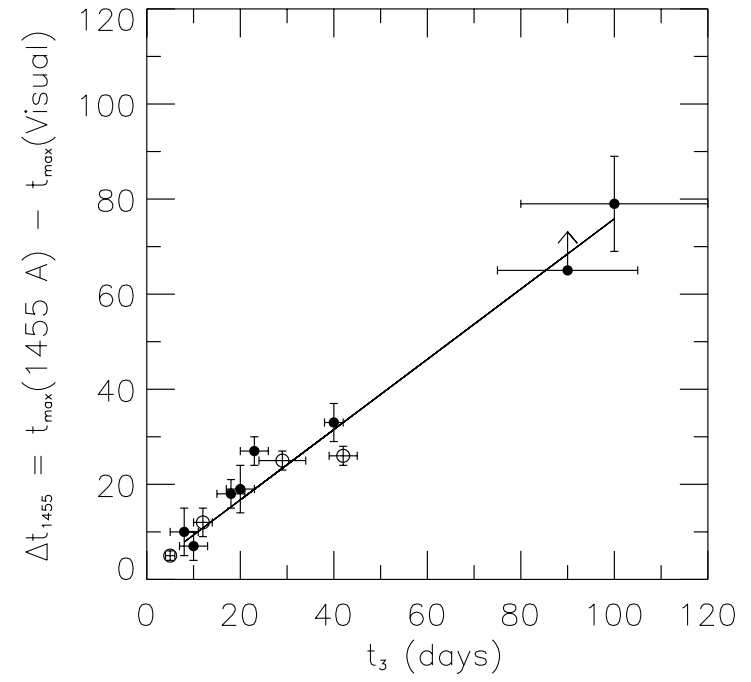

Fig. 4. The time delay $\Delta t_{1455}$ of the flux maximum in the $1455 \AA$ continuum with respect to the visual maximum is plotted as a function of $t_{3}$. Neon novae are indicated as open circles.

way, on several structural parameters, which are often poorly known and sometimes hidden. This may cause individual objects to be seen as peculiar in one or more respects, e.g. in the abundances of the ejecta, the expansion velocities, the energetics, the time scale of the decline, the presence of X-ray or infrared emission etc.

Here, we have concentrated our attention on only one aspect that can be studied quite accurately through the observations, i.e. the time scale of variability of the ultraviolet continuum and of the O I $1300 \AA$ emission line.

In particular, we find that:

- The maximum emission of the $1455 \AA$ continuum lags systematically after the visual maximum, by an amount $\Delta t_{1455}$ which is a linear function of $t_{3}$ (see Eq. (3) and Fig. 4). This behaviour is consistent with the theoretical prediction of a gradual flux redistribution to shorter wavelengths during the constant bolometric luminosity phase shortly following the outburst (Bath \& Harkness 1989; Kato \& Hachisu 1994, and references therein).

- The ultraviolet outburst duration, $\Delta T_{\text {outb }}^{1455}$, increases linearly with increasing $t_{3}$ (see Eq. (4) and Fig. 5).

- The time of the O I $1300 \AA$ maximum emission is a linear function of $t_{3}$ (see Eq. (2)). Being this line excited by hydrogen $\operatorname{Ly} \beta$ photons (see Sect. 3 ), the O I maximum diagnostics the time the ejected envelope starts to be optically thin i.e. the beginning of the transition to the nebular phase.

- The post-outburst progressive hardening of the ultraviolet spectrum (see the initial decrease of the ultraviolet colour index $C(1455-2885)$ in Fig. 1) stops somewhat after the $\mathrm{O}$ I flux reaches a maximum value. The following upturn to redder colours or the colour plateau are ascribed to the emerging contribution from the hydrogen Balmer continuum (see Sect. 3).

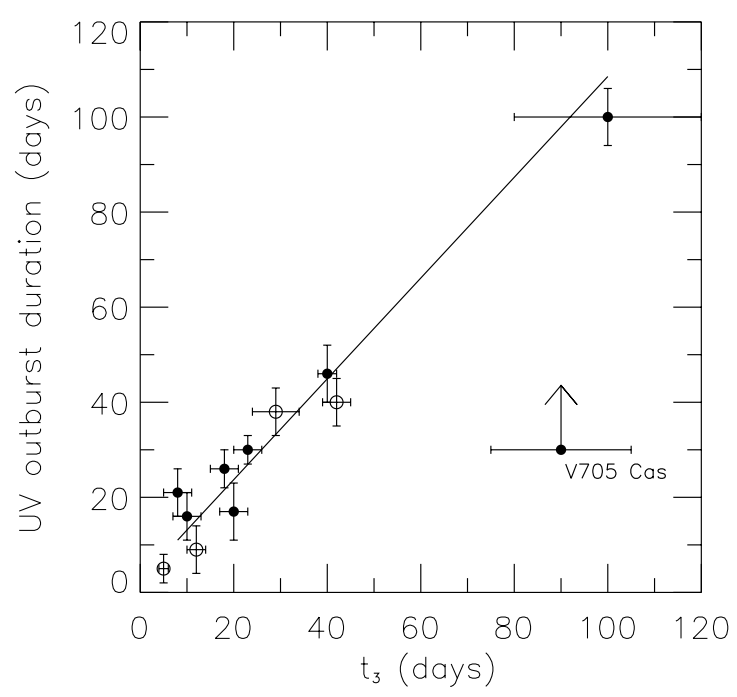

Fig. 5. The duration of the outburst at $1455 \AA$ is plotted as a function of the decay time $t_{3}$. Neon novae are indicated as open circles. The data point for V705 Cas has not been included in the fit.

We have studied the ultraviolet spectra of novae during a time interval that is of the order of $t_{3}$ because during this time the UV continuum and the O I $1300 \AA$ line show the maximum variations. It is remarkable that, within this time interval, none of the observed novae, with the exception of the justified case of the dust forming V705 Cas, shows to be peculiar with respect to the regular patterns discussed above. Not even the more massive Neon Novae (there are 4 in our sample) show any anomaly in this respect. Although it cannot be excluded that a more precise knowledge of the $t_{3}$ times, or a more detailed monotoring in the UV range could allow peculiar objects to be detected at these early outburst stages, the available data are strongly suggestive of a quite homogeneous behaviour insofar the correlation between the ultraviolet and optical variability is concerned.

If $t_{3}$ is taken to be, primarily, an indicator of the white dwarf mass, as discussed by Livio (1992), then the validity of the present results for objects in wide range of $t_{3}$ values, from 5 days for V838 Her to 100 days for PW Vul, suggests that the underlying primary parameter driving the ultraviolet variations in these early phases, is the white dwarf mass.

Indeed, the dependence of the outburst properties on other structural parameters, such as the envelope mass, the expansion velocity and chemical composition of the ejecta, and the temperature of the white dwarf may reveal their influence at later outburst stages. Useful information in this sense can be obtained by studying the dynamics of the ejecta and the long term evolution of the emission lines. Such studies are in progress (Cassatella et al. 2002).

Acknowledgements. This investigation has been financed by contract I/R/70/00 of the Italian Space Agency. We would like to acknowledge Drs. Italo Mazzitelli, Margarida Hernanz, and Vittoria Caloi for interesting discussions. 


\section{References}

Andreä, J., Snijders, M. A. J., \& Cassatella, A. 1991, A\&A, 244,111

Andreä, J., Drechsel, H., \& Starrfield, S. 1994, A\&A, 291, 869

Austin, S. J., Wagner, R. M., Starrfield, S., et al. 1996, AJ, 111,869

Bath, G. T., \& Harkness, R. P. 1989, Classical Novae, ed. M. F. Bode \& A. Evans (J. Wiley, Chichester), 61

Caldwell, J. A. R. 1982, IBVS, 2147

Cassatella, A., Benvenuti, P., Heck, A., et al. 1979, A\&A, 74, L18

Cassatella, A., \& González-Riestra, R. 1990, in Physics of Classical Novae, Lect. Notes in Phys. No. 369, ed. A. Cassatella, \& R. Viotti (Springer-Verlag), 115

Cassatella, A., Altamore, A., González-Riestra, R., et al. 2000, A\&AS, 141, 331

Cassatella, A., Altamore, A., \& González-Riestra, R. 2002, in preparation

Chochol, D., Hric, L., Urban, Z., Grygar, G., \& Papousek, J. 1993, A\&A, 277, 103

Della Valle, M. 1991, A\&A, 252, L9

Duerbeck, W., Rindermann, K., \& Seitter, W. C. 1980, A\&AS, 81, 157

Evans, A., Geballe, T. R., Rawlings, J. M. C., \& Scott, A. D. 1996, MNRAS, 282, 1049

Fitzpatrick, E. L. 1986, AJ, 92, 1068

Gehrz, R. D., Harrison, T. E., Ney, E. P., et al. 1988, ApJ, 329, 894

González-Riestra, R., \& Krautter, J. 1998, ESA SP-413, 367

González-Riestra, R., Cassatella, A., Solano, E., Altamore, A., \& Wamsteker, W. 2000, A\&AS, 141, 343

González-Riestra, R., Cassatella, A., \& Wamsteker, W. 2001, A\&A, 373, 730

Hric, L., Petrik, K., Urban, Z., \& Hanzl, D. 1998, A\&AS, 133, 211

Johansson, S., \& Hamann, F. W. 1993, Phys. Scr., 47, 157

Kastner, A. K., \& Bathia, A. K. 1995, ApJ, 439, 356

Kato, M., \& Hachisu, I. 1994, ApJ, 437, 802

Krautter, J., Ögelman, H., Starrfield, S., Wichmann, R., \& Pfefferman, E. 1996, ApJ, 456, 788

Law, W. Y., \& Ritter, H. 1983, A\&A, 123, 33
Livio, M. 1992, ApJ, 193, 516

Mason, C. G., Gehrz, R. D., Woodward, C. E., et al. 1998, ApJ, 494, 783

Orio, M., Balman, S., Della Valle, M., Gallagher, J., \& Ögelman, H. 1996, ApJ, 466, 410

Orio, M., Covington, J., \& Ögelman, H. 2001, A\&A, 373, 542

Press, W. H., Teukolsky, S. A., Vetterling, W. T., \& Flanney, B. P. 1992, Numerical Recepies (Cambridge Univ. Press)

Robb, R. M., \& Scarf, C. D. 1995, MNRAS, 273, 347

Rodríguez-Pascual, P. M., González-Riestra, R., Schartel, N., \& Wamsteker, W. 1999, A\&AS, 139, 183

Saizar, P., Pachoulakis, I., Shore, S. N., et al. 1996, MNRAS, 279,280

Schwartz, G. J., Starrfield, S., Shore, S. N., \& Hauschildt, P. H. 1997a, MNRAS, 290, 75

Schwartz, G. J., Hauschildt, P. H., Starrfield, S., et al. 1997b, MNRAS, 284, 669

Schwartz, G. J., Hauschildt, P. H., Starrfield, S., et al. 1998, MNRAS, 300, 931

Schwartz, G. J., Shore, S. N., Starrfield, S., et al. 2001, MNRAS, 320, 103

Seaton, M. J. 1979, MNRAS, 187, 73P

Sekiguchi, K., Kilkenny, D., Winkler, H., \& Doyle, J. G. 1989, MNRAS, 241, 827

Shore, S. N., Sonneborn, G., Starrfield, S., González-Riestra, R., \& Polidan, R. 1994a, ApJ, 421, 344

Shore, S. N., Starrfield, S., González-Riestra, R., Hauschildt, P. H., \& Sonneborn, G. 1994b, Nature, 369, 539

Snijders, M. A. J., Batt, T. J., Roche, T. F., et al. 1987, MNRAS, 228, 329

Starrfield, S., Sparks, W. M., \& Truran, J. W. 1986, ApJ, 303, L5

Stickland, D. J., Penn, C. J., Seaton, M. J., Snijders, M. A. J, \& Storey, P. J. 1981, MNRAS, 197, 107

Vanlandingham, K. M., Starrfield, S., Wagner, R. M., Shore, S. N., \& Sonneborn, G. 1996, MNRAS, 282, 563

Vanlandingham, K. M., Starrfield, S., Shore, S. N., \& Sonneborn, G. 1997, MNRAS, 290, 87

Williams, R. E., Ney, E. P., Sparks, W. M., et al. 1985, MNRAS, 212, 753 